\title{
Assessment of Knowledge, Attitude and Practice on Insecticide Treated Net Utilization towards Malaria Prevention among Ethiopian Army members of 24th Division, 2016
}

\author{
Birhane Kidane Gebru ${ }^{1}$, Fasil Kenea Duguma ${ }^{1 *}$ and Worku Tefera ${ }^{2}$ \\ ${ }^{1}$ Department of Public Health, Defense Health Science College, Ethiopia \\ ${ }^{2}$ School of Public Health, Addis Ababa University, Ethiopia
}

Submission: August 26, 2019; Published: September 10, 2019

*Corresponding author: Fasil Kenea Duguma, Defense Health Science College, Department of Public Health, Addis Ababa, Ethiopia

\begin{abstract}
Introduction: Malaria is seasonal in most parts of Ethiopia, with variable transmission and prevalence patterns affected by the large diversity in altitude, rainfall, and population movement. Unstable malaria transmission patterns make Ethiopia prone to epidemics that caused catastrophic public health emergencies.

Methodology: The study was carried out within the army troops of the Western Command at 24th infantry division of three military camps in Kafta Humera, from February to March 2016. It's a quantitative cross-sectional study. 326 study participants were proportionally allocated by systematic sampling method. The sample size was calculated in using single population formula. Data was collected in using face to face interviews. Ethical issue was secured from the concerned institution and study participant. Data would be entered in EPIinfo and analyzed by SPSS. Descriptive and Bivariate analysis was carried out to determine the association.

Result: The ITNs utilization was poor $89(29 \%)$ of 326 members owned ITN. Officer and Non-commission officer, education $>9$ th grade, age $>30$ th, service $>10$ years was identified as significant association with ITNs utilization $(\mathrm{P}<0.05)$.

Conclusion and recommendation: The study participant knowledge $(60 \%)$ and attitude $(86 \%)$ about malaria prevention and control was promising but they had poor practice $(41.7 \%)$ due to this poor risk perception majority of the participant had also, the history of malaria infection before the time of this study. The defense main health department need to give strong attention as major public health issue if it needs to address and fill the gaps highlighted in the study area through systemic, holistic and integrated and persistence health education approach.

Keywords: Malaria; Insecticide treated net; ITN; KAP; Ethiopia; Central command

Abbreviations: EC: Ethiopian Calendar; EMIS: Emergency Management and Integrated Surveillance; FDRE: Federal Democratic Republic of Ethiopia; FGD: Focus group discussion; FMOH: Federal Ministry of Health; HMIS: Health Management Information System; IRB: Institutional Review Board; IRS: Insecticide Residual Spray; ITN: Insecticide Treated Net; KAP: Knowledge Attitude Practice; LLITN: Long Lasting Insecticide Treated Net; MIS: Malaria Indicator Survey; MOP: Malaria Operational Plan; NMCP: National Malaria Control Program; NSP: National Strategic Plan; PPS: Proportionate Population Size; REC: Research and Ethics Committee; SPSS: Statistical Package for Social Science; TNRS: Tgriy National Regional State; U5: Under Five; WC: Western Command; WHO: World Health Organization
\end{abstract}

\section{Introduction}

Malaria is the most prevalent mosquito-borne disease throughout tropical and subtropical regions of the world with huge medical, economic, and social impact [1-4].

According to World Health Organization Report, in 2016, there were 216 million cases of malaria in 91 countries, five million more than the 211 million cases reported in 2015. This marks a return to 2012 levels. Malaria continues to claim a significant number of lives: in 2016, 445000 people died from malaria globally, compared to 446000 estimated deaths in 2015. Children under five are particularly susceptible to malaria. The disease claims the life of a child every two minutes. Fifteen coun- 
tries - all but one in sub-Saharan Africa - carry $80 \%$ of the global malaria burden [5].

The African Region continues to bear $90 \%$ of malaria cases and $91 \%$ of malaria deaths worldwide. Nigeria, the continent's most populous country, accounted for $27 \%$ of malaria cases and $24 \%$ of malaria deaths globally in 2016. As highlighted in the most recent World malaria report, more countries are advancing towards elimination: in 2016, 44 countries had less than 10, 000 cases of malaria, compared to 37 countries in 2010. Since 2010, seven countries have been certified malaria-free (Armenia, Maldives, Morocco, Kyrgyzstan, Sri Lanka, Turkmenistan and United Arab Emirates), and several others are inching closer to obtaining this status [5].

Approximately 4-5 million cases of malaria are reported annually in Ethiopia and the disease is prevalent in 75 percent of the country, putting over 50 million people at risk. Malaria accounts for seven percent of outpatient visits and represents the largest single cause of morbidity. It is estimated that only 20 percent of children under five years of age that contract malaria is treated at existing health facilities [6].

The President of Malaria Initiatives -supported annual micro-planning survey provides even better completeness than both the Public Health Emergency Management and HMIS systems, with essentially $100 \%$ reporting from the 16,003 public health facilities in malicious districts with 57,503 malaria hospitalizations from July 2012 to June 2013 in Ethiopia. There were 4,984,266 total malaria cases, including 4,200,162 laboratory-confirmed and 784,104 presumed (i.e., clinically treated) malaria cases. There were 2,942,031 laboratory-confirmed P. falciparum outpatient malaria cases, and 1,258,131 P. vivax cases. The micro-plan reported 11,132,111 laboratory-confirmed cases out of a calculated total of 11,916,215 suspected malaria cases (suspected cases were formerly termed, "fever cases" per WHO). Although malaria remains the leading cause of Ethiopian outpatient morbidity, and is among the leading causes of inpatient morbidity, it is declining as a relative cause of inpatient mortality, especially among children aged less than five years according to HMIS data [7].

There are about 835 districts with different levels of malaria risk in Ethiopia, with an estimated at- risk population of 50.6 million people as per the new stratification. The best available proxy for local malaria transmission risk in Ethiopia is household altitude below 2,000 meters [7].

According to FDRE, Ministry of Health, the Health-Related Indicator 2015 malaria report showed that Tigray; plasmodium falciparum (15.5\%), plasmodium vivax (11.5\%), Amhara; plasmodium falciparum (32.5\%), plasmodium vivax $(31.2 \%)$; Ben-Gumuz; plasmodium falciparum (10.6\%), plasmodium vivax (6.4\%); Gambela; plasmodium falciparum (1.9\%), plasmodium vivax $(1.3 \%)$. Thus, one of the major preventive methods being the knowledgeable and proper utilization of Insecticide Treated Nets (ITNs) [8].

The Defense Health Main Department (DHMD), since the Ethio-Eritrean war in 1998 has been and still implements a lofty distribution of ITNs and 12 dispenses them to all troops deployed, recruits that have completed their military trainings and peace-keeping troops. Therefore, ITNs distribution is high, however, the malaria prevalence is still also high this indicates a problem of malaria prevention such as ITNs proper utilization of the preventive measures action.

According to the 2015 the division health department malaria by regiment report showed that clinical (11.2\%), malaria confirmed (38.8\%), Plasmodium Falciparum (14.35\%), Plasmodium Vivax (22.5\%), mixed (1.85\%) [9]. Thus, research will focus on the 24th infantry division, located in Kafta Humera, district Tigray it is known that malaria is high. So that, the report indicates malaria cases are high in the army troops, using proper ITNS or not, and the preventive methods are satisfactory. or not there could not scientific study before in this issue. Hence there is a gap inappropriate utilization of ITNs within active military community and this research will focus on KAP in ITN utilization towards malaria prevention will be filling the gap.

Ethiopia has seen a major improvement in LLIN coverage indicators from 2007 to 2015. The Malaria Indicator Survey showed significant the trend in LLIN ownership from 2007 to 2015. The percentage of households in malarious areas owning at least one LLIN is higher in EMIS 2015 (64 percent) than in EMIS 2011 (55 percent), but lower than EMIS 2007 (69 percent) [10].

The finding of 2015 Malaria indicating national survey indicate on average, households in malarious areas own 1.18 LLINs per household. LLIN ownership is slightly higher in urban areas compared to rural areas ( 66 percent versus 63 percent) in the malarious areas. Households in Amhara and Tigray reported the highest LLIN ownership, with 73 percent of them owning at least one LLIN. However, households in Dire Dawa and Harari reported the lowest ownership, with 36 percent and 34 percent of them owning at least one LLIN, respectively. LLIN ownership differed by wealth status, with 73 percent of the households in the fourth quintile owning at least one LLIN, compared to 51 percent in the lowest quintile [10].

Although LLIN ownership is a key indicator for measuring the success of the National Malaria Control Program (NMCP), it is also important in determining if a household has a sufficient number of treated nets for those sleeping within the home. A direct analysis of program impact was made through triangulation of all available malaria data, including use of health facility-based data from newly strengthened surveillance systems. The coverage with LLINs and IRS increased significantly since 2005 to expect substantial program impact. The analysis of available 
evidence suggests that there has been at least a $70 \%$ decline in malaria mortality among children less than five years of age and among older age groups between the years prior to 2005 -when malaria program scale-up began- and 2010. As of 2013, this malaria impact evaluation estimated that there were about 5.3 million annual malaria illnesses and 3,000-6,000 annual malaria deaths $(5 / 100,000)$ among all age groups in Ethiopia, with 1,000 to 2,000 of these occurring among children aged less than five years of age. The updated National surveillance program aims to reduce malaria deaths to about 600-1,000 annually among all age groups $(1 / 100,000)$ by the year 2020 ; this ambitious goal for malaria mortality reduction can only be attained through continued government and donor support and the concerted action among many malaria partners in Ethiopia [7].

Malaria is a major public health problem in Ethiopia despite relatively low malaria prevalence compared to most other malaria-endemic countries in Africa. Unstable malaria transmission patterns make Ethiopia prone to focal and multifocal epidemics that have on occasion caused catastrophic public health emergencies. Malaria is seasonal in most parts of Ethiopia, with variable transmission and prevalence patterns affected by the large diversity in altitude, rainfall, and population movement. Generally, areas located less than 2,000 meters above sea level $(<2,000 \mathrm{~m})$ in altitude are considered malarious areas. The massive scale-up of malaria control interventions, including case diagnosis and treatment, distribution of long-lasting insecticidal nets (LLINs), and indoor residual spraying of households with insecticides (IRS) have preferentially targeted these areas in Ethiopia [10].

Vector control - if well planned, targeted and timely - can contribute significantly in reducing infection risk and saving lives. Vector control is most cost-effective when implemented to prevent an epidemic starting or to introduce control in the very early stages of an epidemic. Vector control is logistically demanding and time-consuming. If delayed or mistimed, its effect can be suboptimal. Th most cost-effective interventions for malaria vector control are IRS and LLINs, if used just prior to, or at the start of, an epidemic (i.e. applied well before the epidemic peak and its subsequent natural decline). Programmes must achieve high coverage to affect transmission [11].

The two major malaria prevention services implemented in Ethiopia are targeted IRS with insecticides and distribution of LLINs for universal coverage. Other vector control activities, mainly larval control through environmental management and chemical larvicide, are also practiced in areas where such interventions are appropriate and expected to have significant impact. The objective of the Ethiopian vector control program is to maintain universal coverage with LLINs and/or have households sprayed with IRS in targeted areas. Based on the new Malaria NSP (2014-2020), stratification and targeting of the LLIN and IRS interventions are being implemented together in high transmission strata to bring down the malaria burden.
The Ethiopian malaria indicate survey (MIS) of 2015 finding in the malarious area indicate, 38 percent of the population where slept under an LLIN the night before the survey. Among people living in households owning at least one LLIN, 61 percent slept under an LLIN the night before the survey. Forty-four percent of pregnant women and 45 percent of children U5 slept under an LLIN the previous night. However, in households owning at least one LLIN, use by children and pregnant women was 70 percent and 74 percent respectively. Knowledge of malaria was higher among women particularly those the highest wealth quintile. Access to LLINs at household level mainly determines use. In addition, community level awareness activities also determine LLIN utilization rates [10].

Overall, 71 percent of households are protected either by owning an LLIN/insecticide-treated net or having received IRS in the past 12 months. Overall, malaria prevalence in Ethiopia is very low. Malaria parasite prevalence in areas $<2,000 \mathrm{~m}$ was 0.5 percent by microscopy blood-slide examination for all ages and 0.6 percent among children U5. Similarly, RDTs indicated the prevalence of infection to be 1.2 percent among all ages and 1.4 percent among children U5 [10].

The Ethiopian army main health department also dispense significant budget on the vector control intervention mainly in providing individual ITN (universal coverage) to each army without including their family (for the family group the ITN was provided from the nearest civilian health office of the resident, insecticide residual indoor spray to each household, bath off (repellant ointment) to all army individually [12].

Despite huge vector control investment still the magnitude of the problem indicate as there is a problem on the transmission and incidence rate and vector control protection factors especially, the prevalence of $\mathrm{P}$. falciparum may indicate existing incidence rate of the new infection. The high magnitude of this incidence may be due to the gap on proper utilization protecting factors (ITN, IRS and bath off repellant), or may be due to lack of access this vector protecting material, outdating effect of protecting factors, outdating and misuse of IRS, maybe due to risk behavior of the army jobs and lifestyle like wearing short shirts, poor risk perception to the problem, poor knowledge, attitude and practice of the protecting and risk factor, mobility behaviors, risky environmental factors like seasonal variation impact on the lifecycle of the mosquito, the infrastructure of drainage, shower, toilet ventilation and illumination of the resident home and plus many other unidentified factor rates.

This study mainly focuses on the risk perception to malaria incidence, awareness and perceived value toward vector control protecting utilization rate and common army related risk factor for malaria incidences. The common reason that motivate us to do this topic in this area was also the observed incidence rate of those new cases, may indicate the existing gap and problem on the issue related with risk factors and disease protection mechanism and capacities. 


\section{Prevention Using Insecticide-Treated Nets}

ITNs have been shown to reduce all-cause child mortality by 17 percent in sub-Saharan Africa and malaria uncomplicated malaria cases among children under five by 50 percent across a range of transmission settings [2]. ITNs also appear to display similar effectiveness under field conditions [3]. Efforts to scale up coverage of ITNs to reach universal use among the population at risk of malaria are underway in most African countries [13].

National Insecticide treated net coverage, ownership, access and presences in Ethiopia from the finding of 2015 Ethiopian malaria indicating survey summarized as follow [10]

Ownership of mosquito nets in the malaria endemic community Ethiopia.

The trend in LLIN ownership from 2007 to 2015. The percentage of households in malarious areas owning at least one LLIN is higher in EMIS 2015 (64 percent) than in EMIS 2011 (55 percent), but lower than EMIS 2007 (69 percent). Ethiopian malaria indicating survey of 2015 indicate, on average, households in malicious areas own 1.18 LLINs per household. LLIN ownership is slightly higher in urban areas compared to rural areas (66 percent versus 63 percent) in the malarious areas. Households in Amhara and Tigray reported the highest LLIN ownership, with 73 percent of them owning at least one LLIN. However, households in Dire Dawa and Harari reported the lowest ownership, with 36 percent and 34 percent of them owning at least one LLIN, respectively.

LLIN ownership differed by wealth status, with 73 percent of the households in the fourth quintile owning at least one LLIN, compared to 51 percent in the lowest quintile. Although LLIN ownership is a key indicator for measuring the success of the national malaria control programme (NMCP), it is also important in determining if a household has a sufficient number of treated nets for those sleeping within the home [9].

The finding of 2015 MIS also shows the percentage of households with at least one LLIN for every two persons who stayed in the household (universal coverage) the night before the interview. Overall, 32 percent of households in the malarious area have reached universal LLIN coverage. Universal LLIN coverage is higher among urban households compared with rural households ( 42 percent versus 30 percent, respectively). Twenty-two percent of households in the lowest wealth quintile have attained universal LLIN coverage, while in all other wealth quantiles over 30 percent of households achieved universal coverage [10].

\section{Access to mosquito nets at National level}

The EMIS 2015 data on access to an LLIN, measured by the proportion of the population that could sleep under an LLIN if each LLIN in the household were used by up to two people. Coupled with mosquito net usage, LLIN access can provide use- ful information on the magnitude of the behavioral gap in LLIN ownership and use, or, in other words, the proportion of the population with access to an LLIN but not using it. If the difference between these indicators is substantial, the program may need to focus on behavior change and how to identify the main drivers/barriers to LLIN use to design an appropriate intervention. This analysis helps the national malaria program determine whether they need to achieve higher LLIN coverage, promote LLIN use, or both [10].

The finding shows percent distribution of the de facto household population by number of LLINs the household owns, according to number of persons who stayed in the household the night before the survey. Thirty-nine percent of Ethiopians living in malarious areas slept in households with at least two LLINs the night before the survey. Twenty-four percent stayed in households that owned one LLIN. About eleven percent of Ethiopians slept in households that owned three or more LLINs. Overall, close to half of the population ( 49 percent) could sleep under an LLIN if each LLIN in the household were to be used by up to two people [10].

As expected, the proportion of persons with access to an LLIN tends to decrease as household size increases. Access to an LLIN is relatively higher for households with two, three, or four persons staying in the household the night before the survey ( 51 -56 percent). LLIN access gradually decreases thereafter [10].

\section{lation}

Use of mosquito nets by households of Ethiopian popu-

Universal coverage of mosquito nets is necessary to accomplish significant reductions in malaria transmission. Moreover, the most vulnerable groups of population, such as children five years of age and pregnant women should be prioritized. The 2015 EMIS asked about the use of mosquito nets by household members during the night before the survey. Fifty-six percent of Ethiopians living in malarious areas were covered by a vector control intervention the night before the survey; that is, they either slept under an LLIN or slept in a dwelling sprayed with IRS in the past 12 months [10].

LLIN use among the general population is higher for children under age 5 years ( 45 percent) and adults 35-49 (43 percent) compared with other age groups. There was no significant difference in sleeping under an LLIN the previous night between females and males. Urban residents (48 percent) are more likely than rural residents (38 percent) to have slept under an LLIN. By region, LLIN use is highest in Afar (51 percent) and Amhara (43 percent), the lowest among people living in and Harari (17 percent) and Dire Dawa (16 percent). LLIN use steadily increases with wealth; 47 percent of those in the fourth wealth quintile compared with 26 percent of those in the lowest wealth quintile slept under an LLIN the previous night [9]. In malarious areas, 62 percent of existing LLINs in households were used the night 
before and the proportion of LLINs used by at least one household member was higher in urban areas than in rural areas (71 percent and 61 percent, respectively) [10].

\section{General malaria knowledge at national level}

In malarious areas, Sixty-eight percent of women in malarious areas have heard about malaria. Of those who had heard of malaria, 75 percent of women reported mosquito bites as a cause of malaria. Knowledge of this was higher among women in the highest wealth quintile (82 percent) than among women in the lowest ( 66 percent). Of those who had heard about malaria, 77 percent knew that sleeping under mosquito nets can prevent malaria. FMOH, partners and stakeholders should apply practical approaches to achieve universal net coverage in the targeted malaria risk areas. Needs an appropriate planning and implementing timely distribution of LLINs to household level.

Access to LLINs at household level mainly determines use. In addition, community level awareness activities also determine LLIN utilization rates. Among those households in malarious areas owning at least one LLIN, the LLINs utilization rates have improved when compared with MIS-2007 and 2011 indicating improvements on community awareness.

National malaria parasite prevalence for all age groups has been declined compared to MIS 2007 and 2011. Malaria prevalence is highest in the age group 35-39 years compared to other age groups. But in the Ethiopian context, MIS is not a good tool to measure malaria prevalence as the malaria burden is very low and seasonal. Malaria prevalence is higher in male and rural residents but no variation with economic status [10].

The high morbidity rate in the adult population significantly reduces production activities of Ethiopian army trainee and community in the endemic malaria area; the prevalence of malaria in many productive parts of the country prevents the movement and settlement of army in resource- rich low-lying river valleys; coping with malaria epidemics overwhelms the capacity of the army health services, and thus substantially increases public health expenditures of Ethiopian army health office. With this reality, the assessment of the common risk factors, risk perception to malaria infection, perceived value and utilization rate of vector control protecting factors (ITN, IRS, Repellant) may help to understand and to have measurable insight about the causes to the existing morbidity rate.

Moreover, monitoring and assessment malaria Bio-behavioral status may have a lot of contribution in reducing the incidence of new case and transmission magnitude, in addition to that such kind of study may have an impact in managing malaria burden due to poor effect of insecticide resistances protecting capacity of ITN and this may have a key Contribution in intensifying the rate of indoor and outdoor resting and biting habit of anopheles mosquito.

\section{Methodology}

\section{Study area}

The study would be conducted in western Command, at 24th division of in three military camps, Baeker, Kaja and Rawyan, Kafta-Humera district, Tigray National Regional State (TNRS), northern Ethiopia. Humera is located about $973 \mathrm{~km}$ from Addis Ababa, $587 \mathrm{~km}$ northwest of Mekelle, the capital city of Tigray, $340 \mathrm{~km}$ northwest of Bahir Dar, the Head Quarter of WC. The bordering is Sudan on the west and Eritrea on the north. Geographically the area lies between $14^{\circ} 17^{\prime} 26^{\prime \prime} \mathrm{N}$ latitude and $36^{\circ}$ $36^{\prime} 29^{\prime \prime}$ E longitude with an elevation varying from $550 \mathrm{~m}$ to 2800 $\mathrm{m}$ above sea level. The annual rainfall in the area ranges from $500 \mathrm{~mm}$ to $611 \mathrm{~mm}$. The mean maximum temperature is high ranging from 300C in August to 400C in March and May. The relative humidity is highest during the months of rain (June-July) and lowest at the end of the dry season in April [13].

Study period: The study would be carried out from February 8, 2016 to March 4, 2016.

Source and study population: The source population includes all the divisions and staff under the Western command. The target population is army members of the Western command.

\section{Inclusion and exclusion criteria}

Inclusion criteria: Military personnel those who were in the age range of 18 - 52 years.

Exclusion criteria: Military personnel those who were not in the age range of 18 - 52 years. Civil servants absent for second visit during data collection period.

\section{Study design}

Quantitative cross-sectional study design.

\section{Variables of the study}

\section{Independent variables}

a) Socio-demographic characteristics: Rank, age, sex, religion, marital status, ethnicity, level of education, service year,

b) Condition of the camp: permanent, temporary, people sleep in one room, sleeping area.

c) Knowledge about malaria: mode of transmission, mosquito breeding sites, prevention methods, Personal protection. Attitude: on prevention method in relation to ITNs utilizations, side effects associated with ITNs utilization, type of ITNs preference.

\section{Dependent variable}

a) KAP on ITN utilization. 


\section{Measurement variable}

Knowledge toward ITN utilization: There were thirteen questions to study the knowledge status of respondents. We gave one score for each correct response. The Possible total score is 13 . Minimum score has 1 (one) point, maximum score has 12 points, mean score has 9 points and knowledge status of respondents was categorized into poor knowledge level (if the knowledge score less than 9 or mean value), good knowledge level (knowledge score above mean value).

Attitude toward ITN utilization: There were six questions used to measure the attitude status of respondents. We gave one score for each correct response. Possible total score has 6 points. Minimum score has 1 (one), maximum score has 6, mean score has 5 points. Most of respondent attitude was categorized in negative attitude level (attitude score less than 5), positive attitude level (attitude score above 5).

Practice toward ITN utilization: There were eight questions to assess the practice level of the respondents. We gave one score for each correct response. Possible total score was 8 . Minimum score was 1 and maximum was 7 . Mean score was 5.5 and. Practice status of respondents was categorized into poor practice level (practice score less than 5), and good practice level (practice score greater than 5) good practices

Data collection instrument and method: Data would be collected by using face to face interview questionnaires. The questions would be initially prepared in English and then translated into Amharic and translated to English again to check its consistency. A questionnaire would be adopted from other similar studies on KAP on ITNs use and determinant factors based on consultations and literature review. The questionnaire has six sections that contain the demographic characteristics, condition of Home, knowledge toward malaria, knowledge about ITNs use, attitude and practice towards malaria prevention. Data would be collected with the help of six trained professionals who have diploma/degree in health-related fields.

Validity and reliability: Content Validity would be ensured by careful assessment and selection of questions from the spe- cific objective point from the piloted WHO malaria assessment tool the final questioner adopted. These questions reflect the concept of knowledge, attitude and practice (KAP) on ITN towards malaria prevention. The questionnaire would also be carried out by trained health personnel data collectors. Before data collection undertaken all data, collectors and supervisors would be trained about the overall objective of the study. The principal would conduct literature review on journal articles and publications addressing the concept of KAP on ITN before carrying out the questionnaire. The questionnaire would be presented for review to a statistician, senior colleagues and the study supervisor to augment the quality of the quantitative research and ensure practicality. This would help in refining the questions for better meaning, clarity and conceptualization. Before the actual data collection, the questionnaire would be pretested on $5 \%$ of the same characteristics population group and the result would not include in the final study because the aim is to check consistency, reliability of each question and to know the amount of time to conduct interview or self-administration and to assess other related problems.

Sample size determination: The sample size would be estimated using a single population proportion formula and calculated as following. $n=(Z \alpha / 2) 2 p(1-p) / d 2$ Where: $-n=$ the minimum sample size required. $\mathrm{P}=$ proportion of consistent ITN use $0.74 \mathrm{D}=$ marginal error $5 \% \mathrm{Z}=1.96=95 \% \mathrm{CI}$. Therefore $\mathrm{n}=$ $1.962 \times 0.74(1-0.74) / 0.052=3.8416 \times 0.74 \times 0.26 / 0.0025=295.6$ $=29.6 \mathrm{n}=296 \times 10 / 100$ of non - respondent $=29.6=30$ Total sam ple size $296+30=326$.

Sampling technique: Total population in the western command, 24th division Humera would be around 2000 troops the population identified for this study based on the eligible criteria. Stratified random sampling would be used to select study unit and Probability Proportion Size (PPS) would be used to allocate the sample size to each regiment. Finally, the study subject would be selected from each regiment by simple random sampling technique.

\section{Schematic Presentation for Sampling Procedure}

(Figure 1)

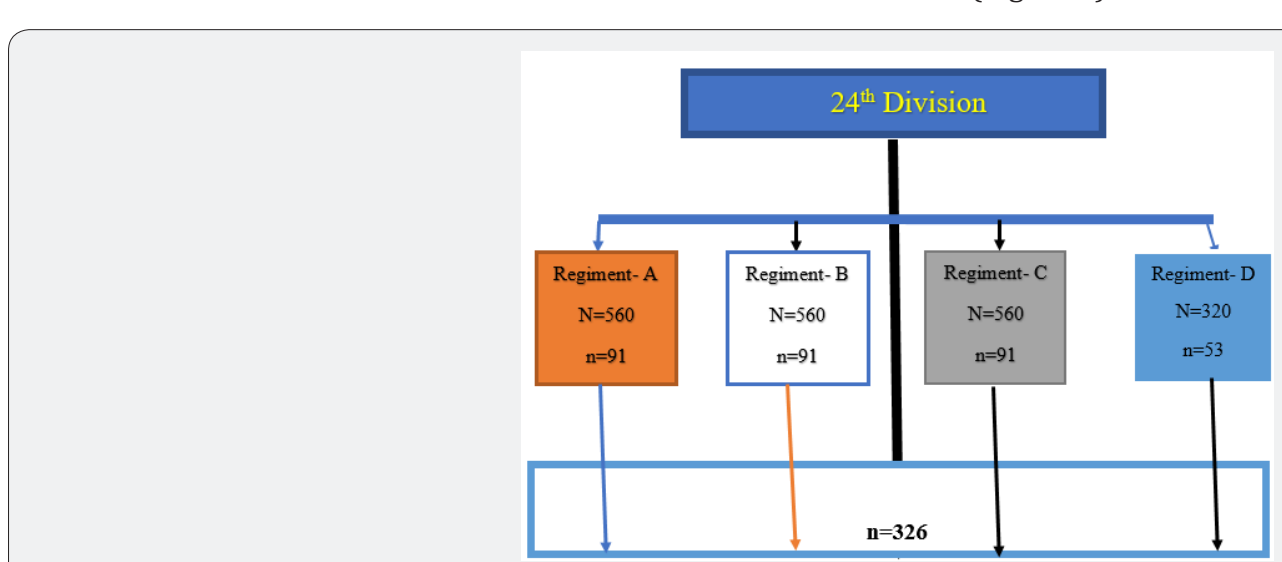

Figure 1: Schematic Presentation for Sampling Procedure. 
Quality assurance: The pre-test was conducted among 5\% of active military personnel to check and secure the reliability and validity issues. The filled questionnaires would be checked and corrected on the spot by principal investigator and supervisor for completeness, inconsistency, uncleared answer and missing values.

Data analysis: Data was cleaned, coded, entered and through EPI Info Version 3.5.1 and analyzed by SPSS Version 20. Bivariate analysis was carried out to determine the association among various variables. Logistic regression was done to control the effects of each dependent and outcome variable. Data was summarized using descriptive statistics including the frequency tables and chi-square. P-Value of less than or equal 0.05 was used as a cut off point for declaring the presence of statistically significant association. Odds ratios and $95 \%$ confidence intervals was computed.

Ethical consideration: Ethical clearance was obtained from Research and Ethics Committee (REC) and Institutional Review Board (IRB) of the Defense university college of health science. A permission will be obtained from Western Command and 24th Command, hummera. A verbal informed consent was obtained from each respondent. The respondents were informed about the study objective, the way they were selected (sampling issues), privacy, anonyms and confidentiality, total time taken, the risk benefits, their autonomy to withdraw and jump unwanted questions while in the participation [14].

\section{Result}

\section{Socio-demographic characteristics of the study}

A total of 326 army members were participated in this study and the response rate was also $100 \%$. Most of the respondents were males $301(92.3 \%)$ and females 25 (7.7\%). Mean age of respondent was $24(\mathrm{SD}+4.74)$. Military rank of participants was 124 (38.3\%), private soldiers,190 (58.0) non-commission officer, and Officer 12 (3.7\%). On the marital status aspect most of the participants were single 271 (83.1\%), 53 (16.3\%) mar- ried and $2(0.6 \%)$ divorced. From the Educational level point of view 76 (23.3\%) grade 7-8, 208 (63.8\%) grade 9-10,33 (10.1\%) Grade $11-12$ and 9 (2.8\%) diploma to degree. Regarding service year most of the respondents $242(74.2 \%)$ joined to the army between 8-16 year, and the remaining 84 (25.8\%) were joined before 16 years. According to the living condition most participants $221(67.8 \%)$ living in temporary military camp, the rest $105(32.2 \%)$ were living in permanent military compound. Majority of the participant 240 (73.6\%) sleep and occupy one room for 6 people, 62 (19\%) were occupy and sleep in room for seven to twelve in one room and 24 (7.4\%) participant sleep for more than thirteen individuals in one room. On the other hand, most of the respondents 221 (67.8\%) sleep on medeb / floor (a kind of bed made from mud)1, the remaining 105 (32.2\%) sleep on the bed.

\section{Source of information about malaria}

Respondents 297 (91\%) replied that they heard about malaria and 29 (8.9\%) said that they were not informed. Majority of respondents 300 (92\%) knew about how malaria was transmitted through mosquito bites however, few respondents replied as malaria is transmitted by bedbug $4(1.2 \%)$, by breathing $4(1.2 \%)$, by touching $9(2.8 \%)$. Regarding the mosquitoes breeding site respondents replied that almost half of respondents by 184 (56.4\%) Stagnant water, thrown old cans 12 (3.7\%), thrown old tire $6(1.8 \%)$, Multiple response (Stagnant water, thrown old can and tire) 119 (36.5\%), poor personal hygiene (0.6\%), don't know $3(0.9 \%)$. The other important information was previous illness of malaria, $83 \%$ of participants were previously infected by malaria this may indicate that the burden of the disease in the study areas. Regarding insecticide Treated net information the study indicated that $326(100 \%)$ of the respondents got information about ITN from different sources. Health personnel (60\%), Mass media (6\%), Colleague (2\%), multiple response of the previse three variables (32 5\%) were major sources of information about ITN. The focus group discussion result indicate as the regiment level one hospital provides health education every month about malaria and ITN utilization (Figure 2).

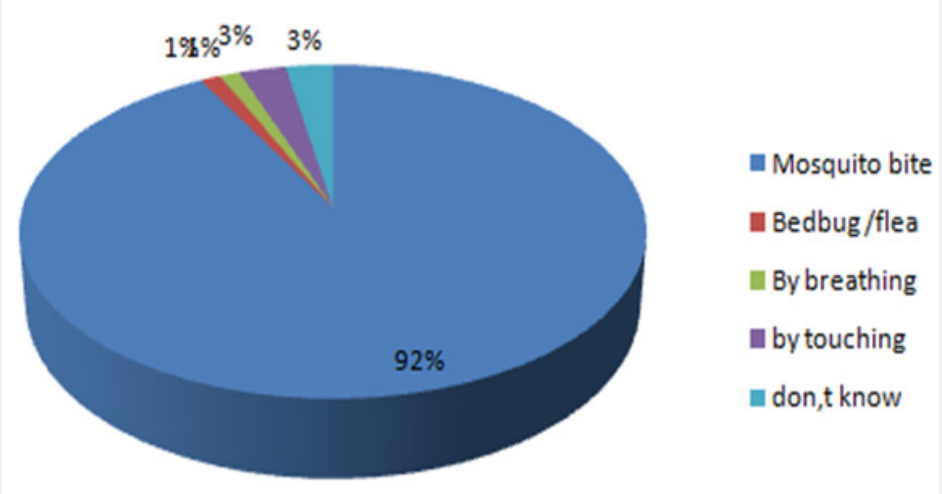

Figure 2: shows Respondents answer towards source of information about ITN. 


\section{Knowledge of respondent toward malaria}

About $8.9 \%$ (29) had not heard about malaria even if the area was malaria endemic. Regarding, breading place of malaria majority of the participant were not able to answer main bread- ing place of malaria, 3.7\% (12) thrown old can, 1.8\% (6) thrown old tire, 36.5\% (119) respond multiple answer either(stagnant water, thrown old tires, thrown old cans) but about $83.7 \%$ (283) had the history of malaria infection before this study (see Table 1).

Table 1: Knowledge of respondents towards malaria in, army troops of the Western Command, at $24^{\text {th }}$ infantry division of in Kafta Humera district, Tigray, Ethiopia, March 2016.

\begin{tabular}{|c|c|c|c|}
\hline \multicolumn{2}{|c|}{ Study Variables } & Frequency(f) & Percentage (\%) \\
\hline \multirow{2}{*}{ Have you ever heard about malaria? } & Yes & 297 & $91 \%$ \\
\cline { 2 - 4 } & No & 29 & $8.90 \%$ \\
\hline \multirow{5}{*}{ Do you know about Mosquitoes Breeding site? } & Stagnant water & 184 & $36.40 \%$ \\
\cline { 2 - 4 } & Thrown old can & 12 & $1.70 \%$ \\
\cline { 2 - 4 } & Thrown old tire & 6 & $36.50 \%$ \\
\cline { 2 - 4 } & Multiple respond & 119 & $0.60 \%$ \\
\cline { 2 - 4 } & Poor personal hygiene & 2 & $0.90 \%$ \\
\cline { 2 - 4 } & Do not know & 3 & $83.70 \%$ \\
\hline \multirow{2}{*}{ Have you ever had an attack malaria? } & Yes & 283 & $16.30 \%$ \\
\cline { 2 - 4 } & No & 53 & \\
\hline
\end{tabular}

Knowledge about malaria prevention method.

a) Among the respondents who aware about malaria prevention methods. The respondent replied using ITNs was 124 (38\%), using Buzz-off 54 (16.6\%), wearing cloths $44(13.5 \%)$, and multiple response 92 (28.2\%) respectively army members respond prevent malaria. Regarding the function ITN participants replied that 124 (38\%) kill mosquito,12 (3.7\%) repel mosquito, 215 (66\%) both repeal and kill mosquitoes.

\section{Level of knowledge about malaria and ITN}

a) The result of this study indicates that $39.6 \%$ (129) of the respondents had poor knowledge and $60.4 \%$ of the respondents had good knowledge. The attitude of the respondent toward ITN utilization

b) Among 326 respondents, 15.6\% (51) had negative attitude and $84.4 \%$ (275) had positive attitude toward ITN utilization.

\section{Practice of the participant toward ITN utilization.}

a) Among the respondent $41.7 \%$ (136) has poor practices on the prevention of malaria and $58 \%$ good practices. The coverage of insecticide-treated bed nets (ITNs) usage in army troops of the Western Common, at 24th infantry division of in Kafta Humera district, the research sought to find out the number of respondents who own ITN were $91.7 \%$, but $50 \%$ of the respondents sleep under ITN regularly.

\section{Discussion}

\section{The study described the Factors Associated with} Knowledge, Attitude and Practice of ITN utilization
a) Rank: Non-commissioner officer 2 more knowledge- able than other ranks (officer and privet army)
b) Participant living condition: soldiers those who live in temporary living house 1.7 time knowledgeable than soldiers who live in permanent living condition $\mathrm{AOR}=1.7$ (95\%CI 1.0-2.7) (See Table 2)

Table 2: Bivariate and multiple logistic regression analysis for factors affecting knowledge on ITNs in Army troops of WC,24 $4^{\text {th }}$ division, Tigray, Ethiopia $2016(n=326)$.

\begin{tabular}{|c|c|c|c|c|c|}
\hline \multirow{2}{*}{ Variables } & \multicolumn{2}{|c|}{ Knowledge Level } & \multicolumn{2}{|c|}{ Odds Ratio (95\% CI) } & \multirow{2}{*}{ P-Value } \\
\hline & Poor & Good & Crude Odd Ratio & Adjusted Odd Ratio & \\
\hline \multicolumn{6}{|l|}{ Military Rank } \\
\hline Private & 62 & 62 & 1 & 1 & \\
\hline None Officer & 63 & 127 & $2.0(1.3,3.2)^{*}$ & $2.0(1.3,3.2)^{*}$ & $\mathrm{P}<0.05$ \\
\hline Officers & 4 & 8 & $2.0(0.6,6.9)$ & $2.1(0.6,7.5)$ & \\
\hline \multicolumn{6}{|l|}{ Living Room } \\
\hline Temporary & 80 & 143 & $1.6(1.0,2.6) *$ & $1.7(1.0,2.7)^{*}$ & $\mathrm{P}<0.05$ \\
\hline Permanent & 49 & 54 & 1 & 1 & \\
\hline
\end{tabular}


c) Military rank: Those solider who are None commissioner officer 2 time use ITN than Private soldiers AOR=1.9(95\% CI 1.2-3.2) and d) Number of inhabitants in the room: The numbers of soldier's room occupancy, those who (1-6) living together 3 time use ITN than who live greater than 13 soldiers. AOR $=3$ (95\% CI 1.2-7.5)

Table 3: Bivariate and multiple logistic regression analysis for factors affecting Attitude on ITNs in Army troops of WC, $24^{\text {th }}$ division, Tigray, Ethiopia, 2016.

\begin{tabular}{|c|c|c|c|c|}
\hline \multirow{2}{*}{ Variable } & \multicolumn{2}{|c|}{ Attitude } & \multicolumn{2}{c|}{ Odds Ratio (95\% Confidence Interval) } \\
\cline { 2 - 5 } & Negative & Positive & Crude odd ratio & Adjusted Odd Ratio \\
\hline Age & & & 1 & 1 \\
\hline $18-23$ & 30 & 129 & $1.7(0.9,3.3)$ & $2.3(1.0,4.9) *$ \\
\hline $24-29$ & 16 & 119 & $1.6(0.4,7.5)$ & $3.5(0.6,21.4)$ \\
\hline $30-35$ & 2 & 14 & $1.0(0.3,3.8)$ & $1.1(0.3,4.9)$ \\
\hline$>36$ & 3 & 13 & 1 & 1 \\
\hline Education Level & 14 & 62 & $1.5(0.7,3.0)$ & $1.4(0.7,2.8)$ \\
\hline $3-8$ grade & 27 & 181 & $1.0(0.4,2.9)$ & $1.0(0.4,3.0)$ \\
\hline -11-12 grade & 6 & 27 & $0.3(0.07,1.2)$ & $0.1(0.03,0.7) *$ \\
\hline
\end{tabular}

Table 4: Bivariate and multiple logistic regression analysis for factors affecting practice on ITNs in Army troops of WC, $24^{\text {th }}$ division, Tigray, Ethiopia, 2016.

\begin{tabular}{|c|c|c|c|c|c|}
\hline \multirow{2}{*}{ Variable } & \multicolumn{2}{|c|}{ Practice } & \multicolumn{2}{|c|}{ Odds Ratio (with $95 \%$ Confidence Interval) } & \multirow{2}{*}{ P- Value } \\
\hline & Poor & Good & Crude Odd Ratio & Adjusted Odd Ratio & \\
\hline \multicolumn{6}{|l|}{ Military Rank } \\
\hline Private & 64 & 60 & 1 & 1 & \\
\hline NCOI & 66 & 124 & $2.0(1.3,3.2)^{*}$ & $1.9(1.2,3.2)^{*}$ & $\mathrm{P}<0.005$ \\
\hline Officer & 6 & 6 & $1.1(0.3,3.5)$ & $0.9(0.3,3.2)$ & \\
\hline \multicolumn{6}{|c|}{ Number of People Sleep in One Room } \\
\hline 1-6 individual & 95 & 145 & $3.1(1.3,7.4) *$ & $3.0(1.2,7.5)^{*}$ & $\mathrm{P}<0.005$ \\
\hline 7-12 individual & 25 & 37 & $2.9(1.1,7.9) *$ & $3.0(1.1,3.4)^{*}$ & $\mathrm{P}<0.005$ \\
\hline$>13$ individual & 16 & 8 & 1 & 1 & \\
\hline
\end{tabular}

e) Participant age between 24-29 were 1.7 times had good attitude than other age group of the participants. College graduate participant had 10 times negative attitude toward INT use than other educational status. (See Table 3 \& 4)

According to the result gained from FGD, the distribution of ITN was mainly from Health department of the division and some of the army members owned ITNs from nearest public health facility, maybe due to the nature occupation or mobility most of the army did not used ITN because the majority of Team and Platoon always deployed and posted to the border of Ethio -Eritrea. "The FGD result also indicates this issue as common trend in many members of the battalion and most of them had attacked by malaria" the major reason to this was stated as negligence to use ITN Tuck and wear short while sleeping and at a duty was mainly due to hotness of the deployment area mostly they did not use ITN and this may cause to be attacked by malaria.
Furthermore, this study shows the association between knowledge, attitude and practice toward ITN Utilization was described as follows

a) The Association between respondents' knowledge and attitude shows that as there was statistically significant association between respondents' knowledge and attitude. ( $x 2=9.372, d f=1 p=0.02$ ). Of those $89.3 \%$ who have good knowledge have positive attitude but those who had $23.3 \%$ poor knowledge have negative attitude toward ITN utilization.

b) The association between respondents' knowledge and practice also indicate as there was statistically significant association between respondents' knowledge and attitude. $(\mathrm{X} 2=13.81 \mathrm{df}=1 \mathrm{p}=0.000)$. Of those $66.5 \%$ who have good knowledge have good on ITN utilization status but $45.5 \%$ those who have poor knowledge have poor utilization of ITN. 
c) The association between respondents' attitude and practice also indicate as there was statistically significant association between respondents' attitude and practice $(\mathrm{X} 2=10.99, \mathrm{df}=1 \mathrm{p}=0.001)$. Of those $62.2 \%$ who have positive attitude have good ITN utilization status but $37.3 \%$ those have negative attitude have also poor utilization of ITN.

\section{Discussion summery of this study (Central Command) in competing with study conducted in wester com- mand which is similar in weather(hot), setup and geographic endemic malaria area of Ethiopian army training centers}

Study conducted among military personnel of 37th Division central command on "the assessment of malaria on knowledge and its associated factor" indicates high self-report of malaria infection rate that is $136(34 \%)$ of participant before three months of the study was infected with malaria, smaller in rate 274 (84\%) than this study, this may indicate the level of malaria risk as the major public health problem in both central and western command.

When it comes to transmission of malaria only 246 (61\%) versus $300(92 \%)$ stated anopheles mosquito as a vector that transmits malaria, about the behavior of biological vector, the majority 361(89.6\%) versus 260 (80\%) stated that it bites during the night, while a small number $42(10.4 \%)$ versus $66(20 \%)$ reported day time and the finding showed that the community's over all awareness about transmission and preventive measures of malaria was good. However, the problem had continued, this issue may be one of the important factors responsible for the consistence of malaria in endemic areas.

In addition, majority of the subjects had high $376(94 \%)$ coverage of ITN like our study $300(92 \%)$ but, practice of protective measures uses of this bed nets were poor $184(46 \%)$ in western and $163(50 \%)$ in central commands, the main reason for this was both where tough Ethiopian army mission area and where is high mobility demanding area both in the day and night time. Both central and west command is the main endemic malaria area but poor risk perception, poor practice of vector control protective material, low awareness on the prevention and transmission still overwhelming the prevalence of malaria in the army community.

The finding of this hyperendemic malaria area, Tigray region, central command was similar with our study western command in terms in setup also, the nature of mission need their own lifestyle like wearing of shorthand closes because of the hot weather condition and risky night traveling responsibility. But the previous history of high malaria infection rate in both areas may state the poor risk perception to malaria infection.

The main job character or nature was responsible as contributing factor for low utilization of protecting factor like ITN, so this was common and major malaria prevention and control factor need a new strategy that fit the nature profession-based prevention approach in terms of enhancing the awareness and risk perception unless the army continue in losing those senior and skillful army from their duty because of repeated malaria infection.

\section{Conclusion and Recommendation}

Based on the above findings the researcher concluded the following key point to improve the strategy of prevention and control against malaria amongst the study subjects of north command, Humera district. The knowledge about malaria prevention and control was generally good, but 130 (39.6\%) were did not know about malaria as prevented by ITN. Therefore, compressive and persistent health education is necessary to address and fill the knowledge gaps highlighted by the study. About 15.6\% participants have negative attitude toward ITN utilization. According to FGD results the distribution of ITN from the Health Main Department has $100 \%$ coverage but almost $84 \%$ of the study participant had the history of malaria incidence and this indicates as there is a negligence toward consistent and proper ITN utilization, so this issue need to be considered boldly as major gap in the study area because, the study finding indicate as the attitude statistically significance with the practice of ITN. The general practice of ITN was $41.7 \%$ which is very poor. Therefore, the battalion leaders and coordinator of the army main health department should need to give attention to enhance the compressive knowledge and attitude to add the value of ITN practice mainly to prevent malaria incidences.

\section{Acknowledgement}

My heart-felt gratitude goes to the Defense University College of Health Science, Public Health department and library staff. We would like to extend our appreciation also for School of Public Health administrative staff, the study participants and officials in the study area for their cooperation and provision of valuable information.

\section{References}

1. WHO (2009) Methods for surveillance of antimalarial drug efficacy. World Health Organization. Geneva, Switzerland.

2. WHO. World Malaria Report 2015, Summary.

3. World Health Organization. Geneva, Switzerland 2016, Malaria report.

4. MOP. Malaria Operational Plan Ethiopia 2012.

5. WHO, World Malaria Day 2018 "Ready to beat malaria”- March 2018.

6. http://www.unicef.org/ethiopia/Malaria.

7. President's Malaria Initiative Ethiopia Malaria Operational Plan FY 2015.

8. FDRE, Ministry of Health, Health Related Indicator 2015 Malaria report.

9. Mistry of National Defense division health department malaria regiment report, 2015 Ethiopia. 
10. Ethiopian malaria indicator survey 2015 report.

11. (2012) Jacob Williams, RTI International, Training Manual on Malaria Entomology for Entomology and Vector Control Technicians (Basic Level).

12. Ethiopian army main health department 2018 malaria report.
13. Tedros Adhanom, et al. (2006) Malaria. In: Yemane Berhane, et al (Eds.), Epidemiology and Ecology of Health and Disease in Ethiopia. Shama books. Addis Ababa.

14. Ethiopian army main health department 2015 malaria report.

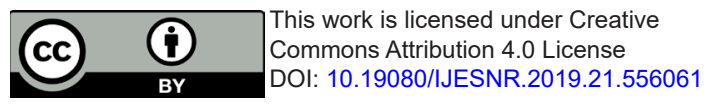

Your next submission with Juniper Publishers will reach you the below assets

- Quality Editorial service

- Swift Peer Review

- Reprints availability

- E-prints Service

- Manuscript Podcast for convenient understanding

- Global attainment for your research

- Manuscript accessibility in different formats ( Pdf, E-pub, Full Text, Audio)

- Unceasing customer service

Track the below URL for one-step submission https://juniperpublishers.com/online-submission.php 